\title{
ILK promotes angiogenic activity of mesenchymal stem cells in multiple myeloma
}

\author{
WEIPENG ZHAO ${ }^{1,2}$, XIAOYING ZHANG ${ }^{1}$, LI ZANG $^{1}$, PAN ZHAO ${ }^{1}$, YAFANG CHEN ${ }^{1}$ and XIAOFANG WANG ${ }^{2}$ \\ Departments of ${ }^{1}$ Breast Cancer and ${ }^{2}$ Hematology, Tianjin Medical University Cancer Institute and Hospital, \\ National Clinical Research Center for Cancer, Key Laboratory of Cancer Prevention and Therapy, Tianjin 300060, P.R. China
}

Received April 21, 2016; Accepted February 6, 2018

DOI: $10.3892 / \mathrm{ol} .2018 .8711$

\begin{abstract}
Angiogenic activity in solid tumors has been demonstrated to promote metastasis through the activation of certain proteins involved in the epithelial-mesenchymal transition-associated process. The molecular mechanism underlying multiple myeloma-induced angiogenesis involves angiogenic cytokines by plasma cells as well as their induction within the microenvironment. Integrin-linked kinase (ILK) is a highly evolutionarily conserved intracellular protein that was originally identified as an integrin-interacting protein, and extensive genetic and biochemical studies have identified ILK expression to be vital during tumor-driven angiogenesis. In the present study, it was identified that angiogenic factors were upregulated in mesenchymal stem cells (MSCs) that were co-cultured with multiple myeloma cell lines. It was also revealed that upregulated ILK expression significantly promoted the capillary-formation ability of MSCs. The concentrations of angiogenic factors were significantly decreased compared with non-targeting siRNA-transfected and control MSCs. MSCs may participate in inducing the angiogenic response in multiple myeloma depending on ILK expression.
\end{abstract}

\section{Introduction}

Multiple myeloma (MM) is the second most common hematological malignancy resulting from the growth of plasma cells within the bone marrow (BM) and is typically accompanied by the secretion of monoclonal immunoglobulins (1). Despite recent progress in treatment modalities, MM is considered to be incurable, but treatable (2). Affecting plasma cells, MM is a hematopoietic malignancy characterized by a peculiar dependency on the bone microenvironment. The interaction of MM

Correspondence to: Professor Xiaofang Wang, Department of Hematology, Tianjin Medical University Cancer Institute and Hospital, National Clinical Research Center for Cancer, Key Laboratory of Cancer Prevention and Therapy, Ti Yuan Bei, Huan Hu Xi Road, Tianjin 300060, P.R. China

E-mail: xiaofangwang2005@163.com

Key words: mesenchymal stem cells, integrin-linked kinase, angiogenesis, multiple myeloma with its microenvironment, including BM stromal cells, osteoblasts, endothelial cells, and cells of the innate and adaptive immune system, including regulatory $\mathrm{T}$ cells, serves a crucial function in the progression, dissemination and drug resistance of MM cells (3). MM cells may reside in the osteoblastic niche for protection from apoptotic stimuli, where they also suppress the formation and function of osteoblasts, leading to impairment of bone formation and the development of osteolytic lesions (4). Improvement in understanding $\mathrm{MM}$ and its microenvironment may result in novel targets for treatment.

Integrin-linked kinase (ILK) was first described as a serine/threonine protein kinase that binds directly to, and transduces signals from, $\beta 1$ integrin subunits, which appeared to require the interaction of integrin cytoplasmic domains with cellular proteins (5). ILK is associated with multiple cellular functions including cell migration, cell proliferation, cell adhesions and signal transduction (6). ILK overexpression was investigated in primary samples and MM cell lines, and the molecular and physiological consequences of small interfering RNA (siRNA)-mediated ILK ablation were compared with treatment with the small-molecule inhibitor QLT0267 (7). ILK was investigated for its potential as a nodal signal integrator for microenvironmental cues in survival pathway activation (7). A number of mechanisms are involved in the crosstalk between stem cells and BM stromal cells, including soluble cytokines, adhesion molecules and certain signaling pathways, including protein kinase $\mathrm{B}$, mammalian target of rapamycin/FK506-binding protein-and rapamycin-associated protein, the Wnt, Notch and parathyroid hormone signaling pathways (7-10).

In a previous study, we investigated the participation of MSCs in inducing the angiogenic response in MM (11). In the present study, the effects of the ILK signaling pathway on MSC-mediated angiogenesis were investigated. It was identified that MSCs induced by MM cells differentiate into smooth muscle cells (SMCs), which depends on the upregulation of ILK expression. Using a co-culture system, an increase in secreted angiogenic factors was identified, which may be involved in MSC differentiation.

\section{Materials and methods}

Cell culture. MM cell lines investigated included RPMI8226 and U266 (American Type Culture Collection, Manassas, 
VA, USA). Ficoll gradients were centrifuged for $40 \mathrm{~min}$ at $400 \mathrm{x} g$ without pause. The bone marrow mononuclear cell layer was then collected, washed in PBS counted and prepared for culture (Invitrogen; Thermo Fisher Scientific, Inc., Waltham, MA, USA). BM cells were cultured at $37^{\circ} \mathrm{C}$ at an initial density of $5 \times 10^{4}$ cells $/ \mathrm{cm}^{2}$ in minimum essential medium- $\alpha$ (Invitrogen; Thermo Fisher Scientific, Inc.) supplemented with $10 \%$ defined fetal bovine serum (DFBS; HyClone; GE Healthcare, Logan, UT, USA), L-glutamine (2 mM; Invitrogen; Thermo Fisher Scientific, Inc.), bFGF (1 ng/ml; R\&D Systems, Inc., Minneapolis, MN, USA) and antibiotic/antimycotic (amphotericin B, penicillin and streptomycin) (Invitrogen; Thermo Fisher Scientific, Inc. \#15240062).

After 1 or 2 days, non-adherent cells were removed and fresh medium replaced the existing culture medium. Medium was changed every 2 or 3 days until reaching confluence. For the co-culture system, $5 \times 10^{4} \mathrm{MSCs}$ were cultured in the conditioned culture medium for 3 days. The supernatant was harvested and added to the MM cultures, which were cultured for a further 1 day. Adherent cells were removed by trypsinization, harvested and cultured by seeding at $1 \times 10^{3}$ cells $/ \mathrm{cm}^{2}$.

Immunophenotyping of MSCs. Following culture of MSCs for 3 days without MM cells until reaching confluence, MSCs were stained with antibodies against cluster of differentiation (CD)11a (\#555380), CD11b (\#553310), CD14 (\#557831), CD29 (\#556047), CD31 (\#560984), CD34 (\#560940), CD44 (\#560977), CD45 (\#560777), CD105 (\#562408), CD106 (\#744313), human leukocyte antigen D-related (HLA-DR; \#562805), CD73 (\#562430), CD90 (\#550402) or matched isotype control (\#562521) (BD Biosciences, Franklin Lakes, NJ, USA), and with anti-CD138 antibody (\#561703, BD Biosciences) to assess potential contamination with residual plasma cells. Immunofluorescence analysis was performed using fluorescence-activated cell sorting (FACS) on a five-parameter flow cytometer (FACS Calibur; BD Biosciences). The FACS protocol was performed as described previously (12).

Preparation of conditioned medium (CM). MSCs were grown in Dulbecco's modified Eagle's medium (DMEM), supplemented with $10 \%$ DFBS and $2 \mathrm{mM}$ L-glutamine. When the cultures were between 80 and $90 \%$ confluent, the medium was exchanged for DMEM without FBS, and CM was collected following 2 days of incubation. CM was filtered through a $0.22-\mu \mathrm{M}$ filter, and used for experiments immediately following preparation.

SMC differentiation. MM cells were fixed directly in a 6-well plate with $4 \%$ formaldehyde for $10 \mathrm{~min}$ prior to being incubated with primary antibodies against $\alpha$-smooth muscle actin ( $\alpha$-SMA; 1:200; cat no. ab21027, Abcam, Cambridge, MA, USA) at $4^{\circ} \mathrm{C}$ overnight. Following incubation with a fluorescein isothiocyanate-conjugated immunoglobulin $\mathrm{G}$ secondary mouse IgG2b antibody or control phycoerythrin for $30 \mathrm{~min}$ at room temperature, (1:150; kit: cat no. 62-6511; Thermo Fisher Scientific, Inc.), washing and resuspension in PBS, flow cytometry analyses were performed with a FACS Aria instrument (BD Biosciences). Analyses were conducted using the CellQuest software (version 5.2.1, BD CellQuest ${ }^{\mathrm{TM}}$ Pro; BD Biosciences) and graphical outputs were obtained using
FlowJo software (FlowJo version 10.0; FlowJo LLC, Ashland, OR, USA).

siRNA transfection. MSCs were transfected with ILK siRNA (\#AM16708, Invitrogen; Thermo Fisher Scientific, Inc) (10 pmol) and irrelevant scrambled [non-targeting (NT)] control siRNA (\#AM4611, Invitrogen; Thermo Fisher Scientific, Inc) using Lipofectamine ${ }^{\mathrm{TM}} 2000$ (Invitrogen; Thermo Fisher Scientific, Inc.), according to the manufacturer's protocol. MSCs at $50 \%$ confluence were treated with siRNA for 1 day in medium lacking serum and antibiotics. Inhibition of ILK protein expression was observed within 1 day, and this knockdown was maintained for at least 3 days following removal of the medium containing the siRNA.

Western blotting. Total protein was extracted using standard protocols using RIPA lysis buffer (\#sc-24948, Santa Cruz Biotechnology). Lysates from whole cell extracts or membrane pellets containing $20 \mu \mathrm{g}$ of proteins were subjected to gel electrophoresis. Proteins were then transferred to polyvinylidene difluoride membranes (EMD Millipore, Billerica, MA, USA). The blots were blocked in 4\% BSA in tris-buffered Tween-20 solution for $30 \mathrm{~min}$ at room temperature and were then incubated at $4^{\circ} \mathrm{C}$ overnight with the primary antibody. The proteins were probed by ILK (1:1,000, \#sc-20019, Santa Cruz Biotechnology), VEGF receptor 2 (1:1,000, \#ab9530, Abcam), $\alpha$-SMA $(1: 1,000$, \#ab21027; Abcam) and $\beta$-actin. After incubation with goat anti-rabbit IgG horseradish peroxidase-conjugated secondary antibodies (1:2,000, \#12-348, Millipore) at room temperature for $1 \mathrm{~h}$, the blot was visualized by ChemiDoc ${ }^{\mathrm{TM}} \mathrm{XRS}+$ System device (Bio-Rad Laboratories, Hercules, CA, USA). Images were analyzed by Image J software (version $1.40 \mathrm{~g}$; National Institutes of Health, Bethesda, MD, USA)

Quantification of angiogenic factors using ELISA. Recombinant vascular endothelial growth factor (VEGF) (\#900-K10), hepatocyte growth factor (HGF) (\#100-39) and basic fibroblast growth factor (bFGF) (\#900-K08) ELISA Development kits (PeproTech, Inc., Rocky Hill, NJ, USA) was used to quantify the angiogenic factors VEGF, HGF and bFGF that were released into the CM of MSCs, according to the manufacturer's protocol.

Formation of tube-like structures. Formation of tube-like structures of MSCs were monitored by light microscopic observation at x100 magnification over six different fields for each well, when MSCs were cultured in CM for 6 days. Custom image recognition and analysis software was implemented in the $\mathrm{C}++$ programming language with the use of the dlib library (http://dlib.net/; date of access, 01/03/2017) for image processing and graphical user interface, Anti-Grain Geometry library (http://www.antigrain.com/; date of access, 01/03/2017) as a vector graphics engine and FFTW library (http://www.fftw.org/; date of access, 01/03/2017) for calculating tube-like structures. Images of the cells were captured for measurement of the length of tube-like structures, as described previously $(11,12)$.

Statistical analysis. All experiments were performed in triplicate. Results are expressed as the mean \pm standard deviation. 
Multiple groups were compared using two-way analysis of variance followed by a Bonferroni's post-test. All statistical analysis was performed by using SPSS (version 11.0; SPSS Inc., Chicago, IL, USA). $\mathrm{P}<0.05$ was considered to indicate a statistically significant difference.

\section{Results}

FACS analysis. The MSC immunophenotype was analyzed using FACS. MSCs expressed CD44, CD105, CD106, CD90, CD73 and CD29, but did not express CD11a, CD11b, CD14, CD31, CD34, CD45 or HLA-DR (Table I).

Angiogenic factors were upregulated in MSCs that were co-cultured with MM cell lines. The classical angiogenic factors VEGF receptor 2 (VEGFR2) and $\alpha$-SMA were identified using FACS (Fig. 1A and B). Importantly, $\alpha-S M A$ was strongly upregulated by co-culture conditions, whereas VEGFR2 was not significantly increased by co-culture with MM cell lines. Co-culture with U266 and PMI8226 myeloma cells appeared to elicit the same results. Angiogenic factors were also detected using western blotting in the two co-culture systems (Fig. 1C and D). In agreement with the FACS results, co-culture of MSCs with the MM cell lines increased $\alpha$-SMA protein expression, but not VEGFR2 expression.

ILK promotes MSC differentiation into SMCs. ILK protein expression was increased in MSC cells co-cultured with U266 and PMI8226 MM cells (Fig. 2A and B). To elucidate whether ILK mediated the effects of MSC differentiation, ILK protein expression in MSCs was knocked down using siRNA. ILK expression was successfully knocked down and $\alpha$-SMA expression appeared to be dependent on ILK (Fig. 2C). These results were investigated further by evaluating the formation of tube-like structures. There was a 5-10\% difference in tube formation ability between knockdown cells compared with controls, while the number of the intersecting branches of assembled cells networks was observed at a magnification of x200 (Fig. 2D). MSCs transfected with ILK siRNA significantly decreased the formation of capillary-like structures in vitro, compared with cells transfected with control and NT siRNA $(\mathrm{P}<0.05$; Fig. 2E)

Quantification of angiogenic factors using ELISA. The levels of the angiogenic factors including bFGF, HGF and VEGF, which were released into the CM of MSCs cultured alone or co-cultured with U266 or RPMI8266, were determined using ELISA (Fig. 3A). All angiogenic factors were significantly increased under co-culture conditions $(\mathrm{P}<0.05$; Table II). The levels of the angiogenic factors were also determined in MSCs following knockdown with ILK siRNA (Fig. 3B). In Table III, bFGF, VEGF and PDGF levels in ILK-knockdown MSCs were significantly decreased compared with NT siRNA-transfected and control MSCs.

\section{Discussion}

Tumor development and progression, which occur in the tumor microenvironment and are characterized by various angiogenic factors, have been demonstrated to be supported
Table I. Immunophenotypes of cultured MSCs.

\begin{tabular}{lc} 
Antigen & $\begin{array}{c}\text { Proportion of MSCs positive } \\
\text { for the antigen, \% (range) }\end{array}$ \\
\hline CD11a & $2.1(0.6-3.4)$ \\
CD11b & $1.3(0.8-2.5)$ \\
CD14 & $3.2(1.1-4.7)$ \\
CD29 & $95.4(91.2-99.8)$ \\
CD31 & $3.1(1.2-4.1)$ \\
CD34 & $2.5(1.1-4.3)$ \\
CD44 & $97.5(93.1-98.5)$ \\
CD45 & $1.9(1.1-3.8)$ \\
CD105 & $97.6(94.4-98.6)$ \\
CD106 & $94.5(91.6-99.1)$ \\
HLA-DR & $3.1(1.3-5.1)$ \\
CD73 & $96.4(91.5-98.2)$ \\
CD90 & $94.1(91.2-99.3)$
\end{tabular}

MSC, mesenchymal stem cell; $\mathrm{CD}$, cluster of differentiation; HLA-DR, human leukocyte antigen D-related.

by angiogenesis. The BM microenvironment in $\mathrm{MM}$ is characterized by an increased microvessel density $(13,14)$. There is growing evidence that integrins serve an essential function in the recruitment of inflammatory cells to tumor sites, and inhibition of integrin function has been suggested as a very promising therapeutic approach for inflammatory diseases and tumors $(8,15,16)$. The pathophysiology of MM-induced angiogenesis is complex, and involves direct production of angiogenic cytokines by plasma cells and their induction within the microenvironment. It has been widely reported that tumor cells produce numerous angiogenic factors, including VEGF, bFGF, epidermal growth factor and matrix metalloproteinases. Previously, the levels of the angiogenic factors bFGF, HGF and VEGF in the conditioned medium of MSCs and the capillary-formation ability of MSCs were determined in vitro (11).

The efficient tumor seeding in a number of, if not all, instances of MM is likely to be supported by the epithelial-mesenchymal transition (EMT) program. Indeed, the induction of EMT suffices to confer on cancer cells an increased tumor-initiating potential and a number of other attributes of MM, including enhanced resistance to chemotherapeutic agents and a lower rate of proliferation (17). Gupta et al (18) reported activation of the ILK/ $\beta$-parvin/cofilin signaling axis is critical to the metastatic colony-forming ability of multiple aggressive cancer cell types.

In the present study, it was demonstrated that ILK may be involved in MSC differentiation into SMCs. MM may induce the secretion of angiogenic factors dependent on the ILK signaling pathway. MM may affect MSCs by direct contact and secreting angiogenic factors. The stromal cells and extracellular matrix have been well-documented to promote the survival of myeloma cells; however, less is known about the influence of MSCs on the angiogenesis of myeloma tumors. In the present study, $\alpha$-SMA was markedly 
A

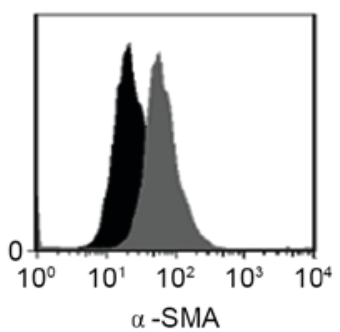

MSCs co-cultured with U266 cells

C

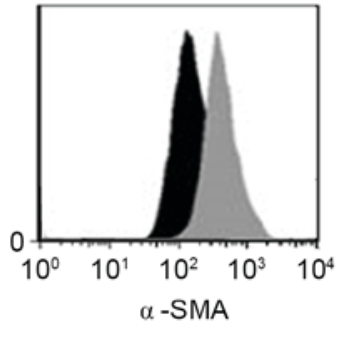

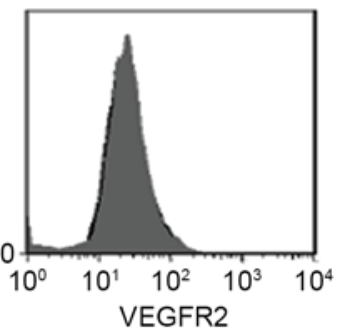

ells

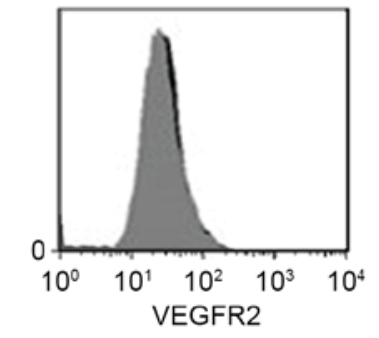

B

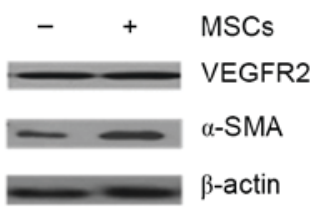

MSCs co-cultured with U266 cells

D

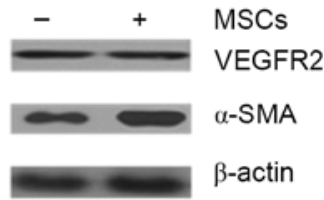

MSCs co-cultured with RPMI 8226

Figure 1. Angiogenic factors were upregulated in MSCs co-cultured with MM cells. (A) VEGFR2 and $\alpha$-SMA protein levels were determined using FACS in MSCs co-cultured with U266 cells for 3 days. (B) VEGFR2 and $\alpha$-SMA protein levels were detected by western blotting in MSCs co-cultured with U266 cells for 3 days. (C) VEGFR2 and $\alpha$-SMA were determined using FACS in MSCs co-cultured with PMI8266 cells for 3 days. (D) VEGFR2 and $\alpha$-SMA protein level were detected by western blotting in MSCs co-cultured with PMI8266 cells for 3 days. MSC, mesenchymal stem cell; MM, multiple myeloma; VEGFR2, vascular endothelial growth factor receptor $2 ; \alpha$-SMA, $\alpha$-smooth muscle actin.

A

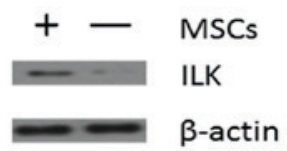

MSCs co-culture with U266

D

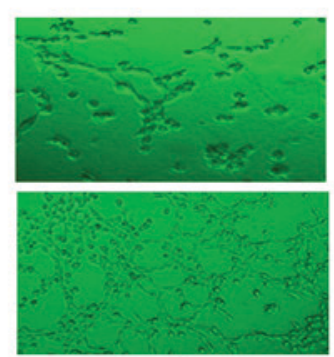

MSCs co-culture with PMI8226
B

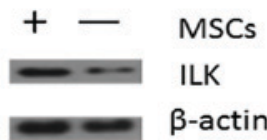

$\beta$-actin
1: ILK-siRNA

$\mathbf{E}$
C

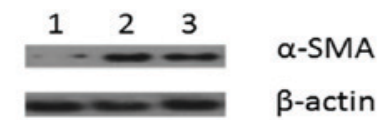

1: ILK-siRNA

2: Non-target siRNA (NT-siRNA)

3: control
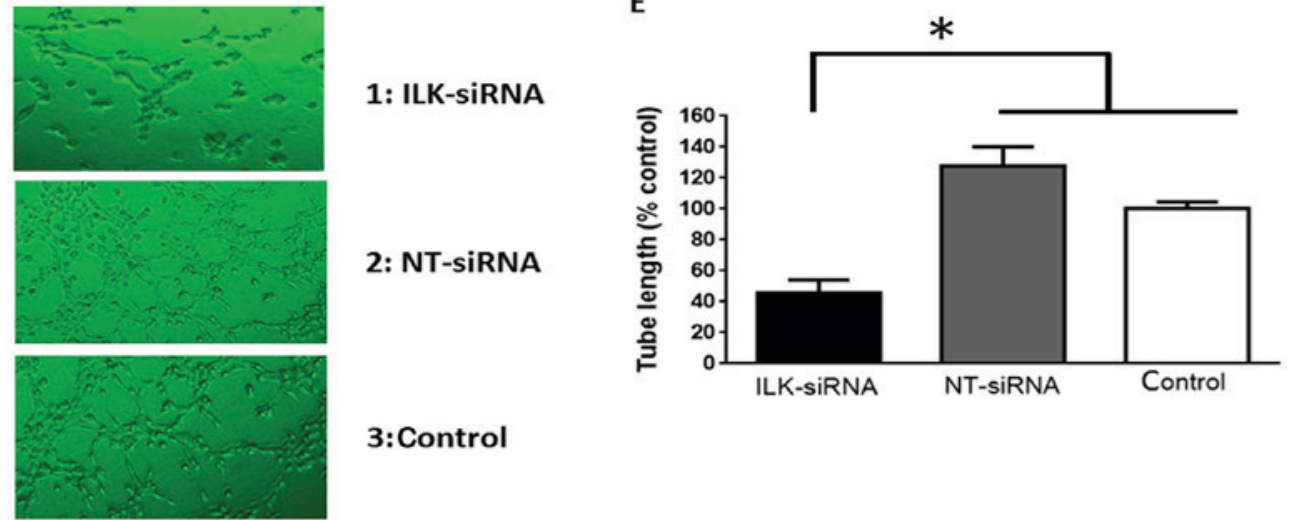

3: Control

\section{C,D,E: MSCs with ILK-siRNA, NT-siRNA and blank control}

Figure 2. ILK promotes MSC differentiation into SMCs. MSCs co-cultured with (A) U266 and (B) PMI8226 multiple myeloma cells for 3 days. ILK protein was detected by western blotting. (C) $\alpha$-SMA protein was detected by western blotting in MSCs transfected with ILK siRNA, NT siRNA or blank control. (D) Formation of tube-like structures of transfected MSCs was determined by microscopic observation at x100 magnification over six different fields of view in each well, when MSCs were cultured in conditioned medium for 6 days. (E) Tube lengths were determined for the transfected MSCs ("P $<0.05$ ). ILK, integrin-linked kinase; MSC, mesenchymal stem cell; SMC, smooth muscle cell; $\alpha$-SMA, $\alpha$-smooth muscle actin; siRNA, small interfering RNA; NT, non-targeting.

upregulated under co-culture conditions, whereas VEGFR2 was not significantly increased by co-culture with MSCs. The repeated western blot results indicated that $\alpha$-SMA was upregulated, whereas further study is required to explain why VEGFR2 is not. $\alpha$-SMA is commonly used as a marker of myofibroblast formation. The presence of pericytes and SMCs 
Table II. ELISA for determination of the concentration of angiogenic factors (bFGF, VEGF and PDGF) in conditioned medium of MSCs in co-culture or cultured alone.

\begin{tabular}{lccc}
\hline Culture & VEGF, pg/ml & PDGF, pg/ml & bFGF, pg/ml \\
\hline MSCs+U266 & $80.41 \pm 7.13^{\mathrm{a}}$ & $121.56 \pm 26.46^{\mathrm{a}}$ & $160.15 \pm 19.53^{\mathrm{a}}$ \\
MSCs+RPMI8266 & $93.25 \pm 8.34^{\mathrm{a}}$ & $89.53 \pm 8.26^{\mathrm{a}}$ & $126.64 \pm 15.67^{\mathrm{a}}$ \\
MSCs & $46.62 \pm 3.41$ & $35.13 \pm 4.23$ & $55.09 \pm 5.13$ \\
\hline
\end{tabular}

${ }^{\mathrm{a}} \mathrm{P}<0.05$. compared with MSC alone. MSC, mesenchymal stem cell; bFGF, basic fibroblast growth factor; VEGF, vascular endothelial growth factor; PDGF, platelet-derived growth factor.

Table III. ELISA for determination of the concentration of angiogenic factors (bFGF, VEGF and PDGF) in conditioned medium of MSCs following siRNA treatment or blank control.

\begin{tabular}{llcr}
\hline Treatment & VEGF, pg/ml & PDGF, pg/ml & bFGF, pg/ml \\
\hline ILK siRNA & $32.23 \pm 2.09^{\mathrm{a}}$ & $17.34 \pm 3.52^{\mathrm{a}}$ & $36.53 \pm 5.21^{\mathrm{a}}$ \\
NT siRNA & $64.56 \pm 7.53$ & $39.96 \pm 5.76$ & $67.45 \pm 6.17$ \\
Control & $71.35 \pm 5.31$ & $47.78 \pm 6.35$ & $73.23 \pm 5.35$
\end{tabular}

${ }^{a} \mathrm{P}<0.05$. compared with untreated MSCs. MSC, mesenchymal stem cell; bFGF, basic fibroblast growth factor; VEGF, vascular endothelial growth factor; PDGF, platelet-derived growth factor; ILK, integrin-linked kinase.

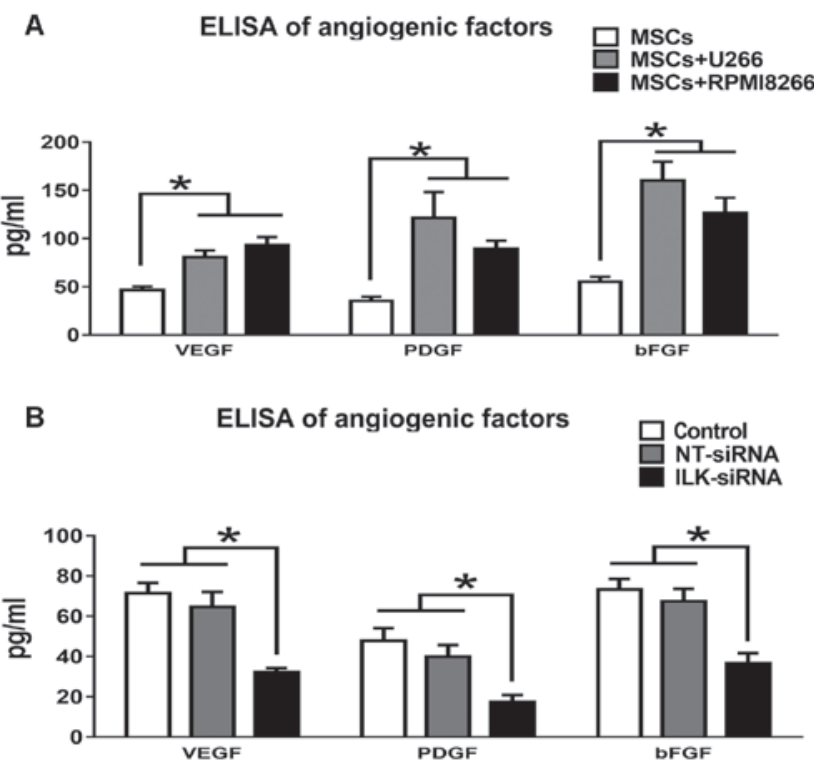

Figure 3. ELISA determination of the concentration of angiogenic factors (bFGF, VEGF and PDGF) in conditioned medium of MSCs. (A) MSCs were co-cultured with U266 or PMI8226 multiple myeloma cells or were cultured alone. (B) MSCs were treated with blank control, NT siRNA or ILK siRNA. "P<0.05. bFGF, basic fibroblast growth factor; VEGF, vascular endothelial growth factor; PDGF, platelet-derived growth factor; MSC, mesenchymal stem cells; NT, non-targeting; siRNA, small interfering RNA; ILK, integrin-linked kinase.

surrounding blood vessels has been described as a structural parameter indicative of vascular maturity. Differentiating fibroblasts express $\alpha$-SMA, indicating the acquisition of a secretory, myofibroblast phenotype, a transition that is associated with increased secretion of profibrotic molecules including collagen and fibronectin (19-21). VEGFR2 appears to be a principal receptor that mediates the mitogenic and chemotactic effects of VEGF on endothelial cells, but it is not clear how. ILK may be associated with $\alpha$-SMA expression. Determination of the underlying molecular mechanism and regulation of ILK requires further study, investigate the efficacy of treatment strategies that directly target the bone microenvironment.

\section{Acknowledgements}

Not applicable.

\section{Funding}

The present study was supported by the National Natural Science Foundation of China (grant nos. 81272562 and 81472183).

\section{Availability of data and materials}

The datasets generated during and analyzed during this study included in the published article are available from the corresponding author on reasonable request.

\section{Authors' contributions}

WZ and XZ were responsible for data collection and interpretation. LZ also participated in data collection. PZ analysed the data and YC performed the statistical analysis. XW was responsible for application of funding, designed the present study, and prepared and revised the manuscript. 


\section{Ethics approval and consent to participate}

The Institutional Ethics Committee of Tianjin Medical University Cancer Institute and Hospital (Tianjin, China) approved the present study.

\section{Consent for publication}

Written informed consents for the publication of related figures had been obtained from the study participants.

\section{Competing interests}

The authors declare that they have no competing interests.

\section{References}

1. Rollig C, Knop S and Bornhäuser M: Multiple myeloma. Lancet 385: 2197-2208, 2015.

2. Quarona V,Ferri V,Chillemi A,Bolzoni M,ManciniC,ZaccarelloG, Roato I, Morandi F, Marimpietri D, Faccani G, et al: Unraveling the contribution of ectoenzymes to myeloma life and survival in the bone marrow niche. Ann N Y Acad Sci 1335: 10-22, 2015.

3. Kikuchi J and Furukawa Y: The mechanisms of drug resistance via the interaction of myeloma cells with stromal cells. Nihon Rinsho 73: 57-61, 2015 (In Japanese).

4. Toscani D, Bolzoni M, Accardi F, Aversa F and Giuliani N: The osteoblastic niche in the context of multiple myeloma. Ann N Y Acad Sci 1335: 45-62, 2015.

5. Hannigan GE, McDonald PC, Walsh MP and Dedhar S: Integrin-linked kinase: Not so 'pseudo' after all. Oncogene 30 4375-4385, 2011.

6. Cabodi S, del Pilar Camacho-Leal M, Di Stefano P and Defilippi P: Integrin signalling adaptors: Not only figurants in the cancer story. Nat Rev Cancer 10: 858-870, 2010.

7. Steinbrunn T, Siegmund D, Andrulis M, Grella E, Kortüm M, Einsele H, Wajant H, Bargou RC and Stühmer T: Integrin-linked kinase is dispensable for multiple myeloma cell survival. Leuk Res 36: 1165-1171, 2012.

8. Shibue T, Brooks MW and Weinberg RA: An integrin-linked machinery of cytoskeletal regulation that enables experimental tumor initiation and metastatic colonization. Cancer Cell 24 481-498, 2013.
9. Song SW, Chang W, Song BW, Song H, Lim S, Kim HJ, Cha MJ, Choi E, Im SH, Chang BC, et al: Integrin-linked kinase is required in hypoxic mesenchymal stem cells for strengthening cell adhesion to ischemic myocardium. Stem Cells 27: 1358-1365, 2009.

10. Goessler UR, Bugert P, Bieback K, Stern-Straeter J, Bran G, Hörmann $\mathrm{K}$ and Riedel F: Integrin expression in stem cells from bone marrow and adipose tissue during chondrogenic differentiation. Int J Mol Med 21: 271-279, 2008.

11. Wang X,Zhang Z and Yao C: Angiogenic activity of mesenchymal stem cells in multiple myeloma. Cancer Invest 29: 37-41, 2011.

12. Janeczek Portalska K, Leferink A, Groen N, Fernandes H, Moroni L, van Blitterswijk C and de Boer J: Endothelial differentiation of mesenchymal stromal cells. PLoS One 7: e46842, 2012.

13. Wang $X$, Zhang $Z$ and Yao C: Bortezomib inhibits the angiogenesis mediated by mesenchymal stem cells. Cancer Invest 30: 657-662, 2012.

14. Ribatti D, Basile A, Ruggieri S and Vacca A: Bone marrow vascular niche and the control of angiogenesis in multiple myeloma. Front Biosci (Landmark Ed) 19: 304-311, 2014.

15. Vacca A, Ria R, Reale A and Ribatti D: Angiogenesis in multiple myeloma. Chem Immunol Allergy 99: 180-196, 2014.

16. Mao Q, Lin CX, Liang XL, Gao JS and Xu B: Mesenchymal stem cells overexpressing integrin-linked kinase attenuate cardiac fibroblast proliferation and collagen synthesis through paracrine actions. Mol Med Rep 7: 1617-1623, 2013.

17. Malinin NL, Pluskota $E$ and Byzova TV: Integrin signaling in vascular function. Curr Opin Hematol 19: 206-211, 2012.

18. Gupta PB, Onder TT, Jiang G, Tao K, Kuperwasser C, Weinberg RA and Lander ES: Identification of selective inhibitors of cancer stem cells by high-throughput screening. Cell 138: 645-659, 2009

19. Shibue T, Brooks MW, Inan MF, Reinhardt F and Weinberg RA: The outgrowth of micrometastases is enabled by the formation of filopodium-like protrusions. Cancer Discov 2: 706-721, 2012.

20. Elzamly S, Agina HA, Elbalshy AE, Abuhashim M, Saad E and Abd Elmageed ZY: Integration of VEGF and $\alpha$-SMA expression improves the prediction accuracy of fibrosis in chronic hepatitis C liver biopsy. Appl Immunohistochem Mol Morphol 25: 261-270, 2017.

21. Tonino $\mathrm{P}$ and Abreu C: Microvessel density is associated with vegf and alpha-sma expression in different regions of human gastrointestinal carcinomas. Cancers (Basel) 3: 3405-3418, 2011. 